\title{
Commentary
}

\section{Treatment Options in Drug Recalcitrant Epilepsy for Physicians: A Brief Introduction to Epilepsy Surgery}

\author{
Sibhi Ganapathy, Rajesh Nair \\ Department of Neurosurgery, Kasturba Medical College \& Hospital, Manipal University, Manipal, India \\ Email address: \\ sibhig09@gmail.com (S. Ganapathy),neurodoc31@gmail.com (R. Nair)
}

\section{To cite this article:}

Sibhi Ganapathy, Rajesh Nair. Treatment Options in Drug Recalcitrant Epilepsy for Physicians: A Brief Introduction to Epilepsy Surgery. International Journal of Neurosurgery. Vol. 4, No. 2, 2020, pp. 22-33. doi: 10.11648/j.ijn.20200402.12

Received: May 13, 2020; Accepted: June 22, 2020; Published: July 28, 2020

\begin{abstract}
Epilepsy surgery has been rapidly progressing over the last decade or so, with many previously untreatable conditions now satisfactorily managed to allow improved quality of life and seizure control without excessive medication and debilitating neurological deficits. We present a brief recap on accepted axioms of epilepsy surgery with a look at the future with a short summary of seizure disorder itself and the various management options. The article is directed at general physicians and Internalists who deal with epilepsy on a daily basis. Many have been exasperated by drug recalcitrant epilepsy and have tried with limited success to manage the condition medically. Many are not aware of the speciality of epilepsy surgery, which as mentioned above has made great strides in seizure control over the last decade. The piece also adds emphasis on diagnosis, seizure semiology and detection along with post procedure analysis and evaluation of efficacy. This has been added to assist the general physician in assessing the disease appropriately and referring the disease correctly to ensure proper diagnosis and treatment early. The authors hope that this piece helps sensitise the physicians to the existence of epilepsy surgery, so that they will refer their patient more and more to neurosurgeons for better care and results to their patients.
\end{abstract}

Keywords: Drug Recalcitrant Epilepsy, Intractable Epilepsy, Epilepsy Surgery, Temporal Love Epilepsy

\section{Introduction}

Epilepsy surgery has been the ugly sister of neurosurgery's charmed souls vascular and skull base surgery. But in the last decade or so, it has occupied an enviable niche for drug recalcitrant epilepsy especially in children. We explain the basic concepts of Epilepsy surgery along with suitable examples below.

\section{Indications}

Patients with refractory epilepsy are generally suitable for one or more procedure. The aim is to either enable seizure free survival or marked decrease in seizure frequency and intensity enabling better quality of life. [1, 2] Epilepsy which is disabling in any way is a candidate for invasive investigations and eventual surgery. All that is expected is an acceptable risk to benefit ratio. $[2,3]$.

\section{Contraindications}

There are a few diseases that are not suitable for surgery. These include disorders such as Idiopathic age-related epilepsies, Progressive underlying neurological diseases, infectious causes, severe mental retardation, and psychopathology. [2-5] An incapability to comprehend and cooperate with the procedure can also be problematic as some procedures require the patient to stay away during surgery and cooperate in an intimate manner. [3] Bilateral, independent inter ictal and/or ictal EEG involvement also makes surgery difficult. Involvement of eloquent cortex by even benign lesions makes surgery difficult as post op deficits become unavoidable. $[4,5]$ Unrealistic expectations at the end of the day are the most difficult and painful obstacle to surmount in the charge to improve quality of life. [6]. 


\section{Intractability}

Stringent criteria for medical intractability as defined are:

(i) failure of two appropriate anticonvulsants,

(ii) the occurrence of an average of one seizure per month for at least 18 months, and

(iii) no more than 3 months of seizure-free hiatus during those 18 months. [5-8]

\subsection{Surgically Remediable Conditions}

Surgery has traditionally been recommended for tumors precipitating epilepsy. [1] The definition has been expanded to include, vascular lesions such as cavernomas, Arteriovenous malformations, aneurysms and hemangioblastomas, developmental malformations such as hemimegaloencephaly, cortical dysplasia's, porencephaly, lissencephaly and others, scar epilepsy, infectious lesions such as abscesses, empyema and encephalitis, strokes and other ischemic insults, and finally traumatic injuries. $[8,9]$ The lesions are summarised in Figure 1, where epilepsy has been divided into Temporal, Extratemporal, and Generalised epilepsy (also called Catastrophic epilepsy) [9].

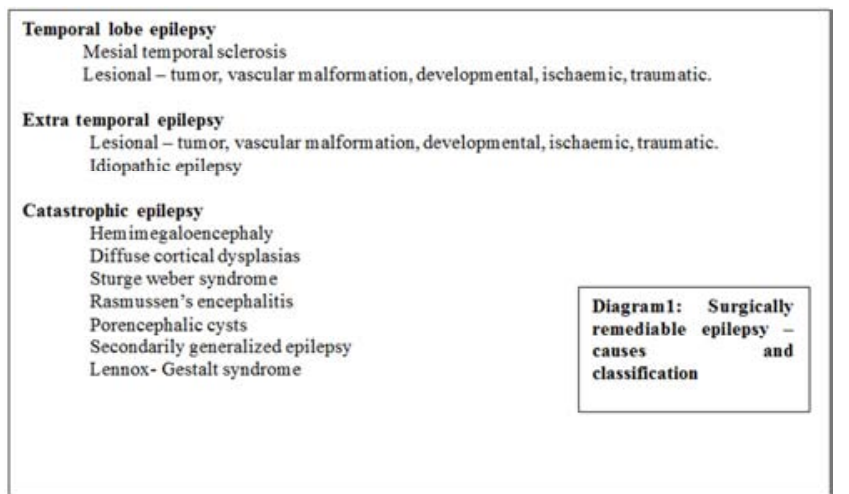

Figure 1. Infectious lesions.

\subsection{Surgical Options}

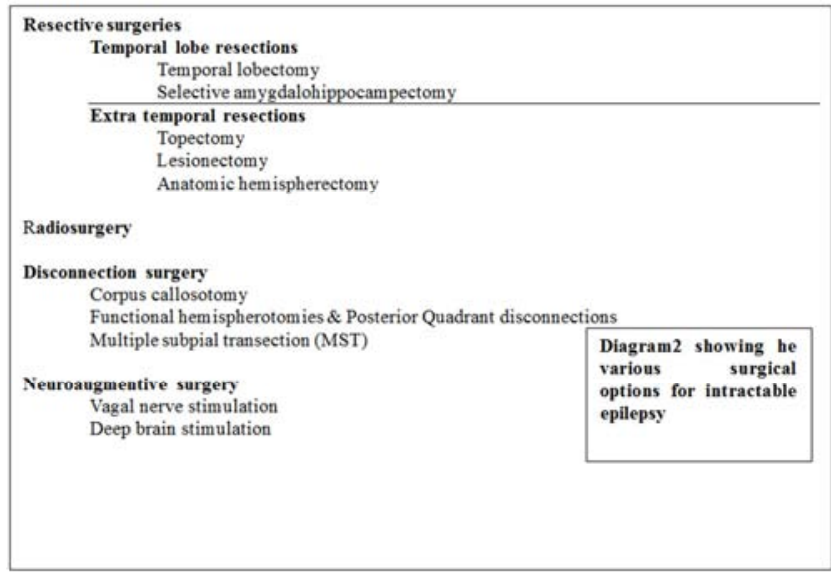

Figure 2. Classified surgery.

Surgery can be classified as listed in Figure 2. There can be resective options such as topectomies, lesionectomies and lobectomies, disconnections such as calosotomies, hemispherotomies, Neuroaugmentative surgery such as anterior thalamic nucleus DBS and vagal stimulation and finally radiosurgery. [10-13]

\section{Patient Evaluation}

All patient evaluation must be done holistically as a multidisciplinary team approach. Diligent an intensive evaluation is essential to ensure good outcomes and prevent unnecessary surgery. Investigation is divided into 2 phases: I and II as shown in Figure $3[12,13]$.

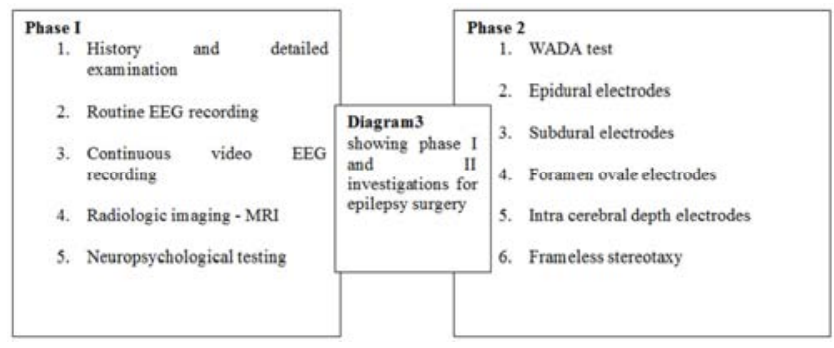

Figure 3. Intensive patient evaluation.

\subsection{Phase 1}

This phase includes non-invasive investigations to attempt localisation of the epileptic focus over the surface of the brain before deciding upon surgery and approach. The phase consists of $[12,13]$.

\subsubsection{History \& Examination}

An accurate history and examination provide a window into the semiology of the disease. Important points to ask and investigate include Auras, Seizure onset, progression, completion, Aphasias, manual automatisms, any and all neurological deficits and lastly neurocutaneous syndromes. 2 or more semiologies would be possible if a multifocal epilepsy is present. Localisation of the seizure focus based on Language (table 2), Autonomic phenomena (table 3), Motor symptoms (table 4) and examination findings (table 5) are summarised as stated. [13, 14].

Seizure semiology

Clinical semiology is often divided into temporal and extratemporal epilepsy. Of the extratemporal group, the frontal lobe stands out as the next most common and diverse group as far as seizures are concerned. We have highlighted the important clinical features of both below.

i. Temporal lobe seizures

These consist of unilateral dystonic posturing of an arm is classical of contralateral temporal lobe epilepsy. Ipsilateral upper arm automatism, postictal coughing with olfactory auras which are poorly recognizable and associated with an unpleasant foul smell ("uncal" or "uncinate" fits). Fear is another limbic aura considered to be amygdaloid in origin. [15]

Patients with left temporal lobe seizures cannot read normally post-ictally. These is associated post-ictal dysphasia, behavioural aggression, sexual features, fetishes, and 
hypergraphia. Temporal Lobe seizures can be separated into Limbic seizures arising from the amygdala (80\%), antero-infero-mesial seizures arising from the entorhinal cortex \& hippocampal area and neocortical seizures arising from the neocortex. The auditory illusory auras may originate from either side of temporal neocortex. [15]

ii. Frontal Lobe Seizures

These are much briefer than their temporal lobe counterparts and are associated with less post-ictal confusion but are more motor in their characteristics. They are more likely to be involved with secondary generalization but are less likely to demonstrate psychic, emotional or other affective phenomena. [15-17] Frontal epileptics are more likely to exhibit a rapid onset and offset and are more likely to occur nocturnally than other partial seizures. The automatisms of frontal lobe are of a "forced nature" These collectively stand in particular contrast to temporal lobe seizures. Frontal seizures can be classified anatomically into Fronto-polar seizures, the origins are often from scars following head injuries. [16] They have the greatest likelihood of simply being characterized by what appear clinically to be primary generalized seizures. They may have some of the general characteristics of FLE, especially contralateral head deviation, Orbitofrontal seizures where patients may have a semiology that mimics temporal lobe seizures in which case the origin is usually attributed to the posterior part of the orbital cortex. Once again, however, it may involve, in various combinations, other frontal lobe semiology, especially contralateral head and eye deviation, Premotor seizures where the epileptiform area includes the supplementary motor area (SMA) Prominent leg movements favour involvement of the supplementary motor area. [18, 19] Head and eye deviation with varying bilateral tonic posturing is the commonest seizure. When the deviation occurs at the onset of the seizure when consciousness is intact it has significant localizing value to the contra lateral frontal cortex. Those seizures arising in the mid part of the frontal lobe have a prominent bilateral tonic posturing. Bipedal automatisms may take the form of symmetric bicycling or kicking movements. [18]

The so-called "fencing posture" is classically associated with the contralateral FL, particularly the SMA. This complex posture is characterized by abduction, external rotation, and partial flexion of the contralateral arm at the shoulder, contralateral deviation of the head and eyes such that they "look at" the contralateral arm, extended ipsilateral arm downwards and backwards, and with the feet apart so as to support the partially contralaterally rotated trunk. Occasionally, the upper limb is also flexed at the elbow with the hand raised to the face that has turned forcefully towards it. [19-21] There is occasionally guttural, ill-understood speech, Dominant opercular seizures where these usually begin with an alteration in speech. The alteration may be typical dysphasic speech, a form of non-specific guttural speech, or an arrest of speech. Post-ictal dysphasia is commonly seen. And finally Rolandic Seizures which combine both motor and sensory components. The sensory features can be positive (e.g. pins and needles, pain, pricking, tingling) or negative (numbness). Elementary paraesthesiae are reported to be the most characteristic of seizures arising in the post-central gyrus. In pure sensory seizures there is nearly always dysfunction of the part involved, which may be awkwardness, typical sensory ataxia, or paralysis. The spread (intracortically) within the Rolandic cortex, e.g., sensory and/or motor, over contiguous parts of the associated homunculus, clinically is reflected in a "march" from one place on the body to another. [21]

Unlike the tonic activity associated with seizures arising outside of the Rolandic cortex, which often has unreliable localizing and lateralizing value, Rolandic motor activity is clonic and is unambiguously localizing to the contralateral motor cortex in the area of the homunculus from which it arises, and usually is associated with transient post-ictal weakness in the involved part $[22,23]$.

The differences between temporal and frontal seizures are summarised in table 1. Apart from history \&clinical examination, investigations form an important part of the phase 1 test battery. These include.

Table 1. DIFFERENCES BETWEEN FRONTAL \& TEMPORAL LOBE EPILEPSY.

\begin{tabular}{|c|c|c|}
\hline FEATURES & FRONTAL LOBE & TEMPORAL LOBE \\
\hline Seizure frequency & Frequent, Often daily & Infrequent \\
\hline Sleep activation & Characteristic & Less common \\
\hline Seizure onset & Abrupt \& explosive & Slower \\
\hline Progression & Rapid & Slower \\
\hline Initial motion/ startling & Less common & Common \\
\hline Automatism & Less common & More common \& Longer \\
\hline Complex posture & Early, frequent \& prominent & Late, Less frequent \& less prominent \\
\hline Hyperkinetic motor signs & Common & Rare \\
\hline Somatosensory symptoms & Common & Rare \\
\hline Speech & Loud vocalization (Grunting, screaming, moaning) & Verbalization speech in non-dominant seizure \\
\hline Seizure duration & Brief & Longer \\
\hline Secondary generalization & Common & Less common \\
\hline Post ictal dysphasia & Rare, unless it spreads to the dominant lobe & Common in dominant lobe \\
\hline
\end{tabular}


Table 2. SPEECH AS A LOCALISING FACTOR IN EPILEPSY.

\begin{tabular}{ll}
\hline SPEECH PATTERN & LOCALISATION \\
\hline Ictal speech arrest & Temporal lobe seizures, usually dominant hemisphere \\
Ictal speech preservation & Temporal lobe seizures, usually non-dominant hemisphere \\
Post ictal dysphasia & Dominant hemisphere involvement \\
\hline
\end{tabular}

Table 3. AUTONOMIC FEATURES OF SPECIFIC SEIZURE SYNDROMES

\begin{tabular}{ll}
\hline AUTONOMIC FEATURES & LOCALISATION \\
\hline Ictus emeticus & Right temporal seizures \\
Ictal urinary urge & Right temporal seizures \\
Piloerection & Left temporal sezires \\
\hline
\end{tabular}

Table 4. MOTOR FEATURES OF SEIZURE SYNDROMES.

\begin{tabular}{ll}
\hline MOTOR FEATURES & LOCALISATION \\
\hline Early non-forced head turn & Ipsilateral of seizure origin side \\
Late forced head turned & Contralateral of seizure origin side \\
Eye deviation & Contralateral of seizure origin side \\
Focal clonic jerking & Contralateral of seizure origin side (peri Rolandic) \\
Asymmetric clonic ending & Contralateral of seizure origin side \\
Dystonic limb posturing & Contralateral of seizure origin side \\
Fencing posture & Contralateral frontal lobe (supplementary motor area seizure) \\
Figure of 4 sign & Contralateral to extended limb, usually temporal lobe \\
Unilateral ictal paresis & Contralateral of seizure origin side \\
Postictal Todd's palsy & Contralateral of seizure origin side \\
\hline
\end{tabular}

Table 5. FOCUSSED EXAMINATION IN EPILEPSY AND ITS SIGNIFICANCE.

\begin{tabular}{ll}
\hline EXAMINATION STEP & SIGNIFICANCE \\
\hline Response to communication & Level of awareness \\
Speech (naming, reading) & Dominant hemispheric involvement \\
Memory (presenting words or phrases for later recall) & Temporal lobe involvement \\
Distractibility & Frontal lobe involvement \\
Response to passive eye opening & To exclude a pseudo seizure \\
Response to physical stimulation & Attention, motor dysfunction \\
Weakness or lack of motor control & Contralateral seizure origin \\
Plantar extensor response & Post ictal paresis \\
\hline
\end{tabular}

\subsubsection{EEG (Electro Encephalogram)}

This is probably the most important investigation that separates pseudo seizures from true epilepsy. It is Positive in $50-60 \%$ of patients with epilepsy. The importance of EEG lies in its ability to:

1. Diagnose epilepsy.

2. To localize the site (using Ictal EEG recordings).

3. To delineate the epileptogenic zone (using Inter ictal EEG).

An Ideal candidate would have a well localized IED's supporting presence of one EZ. Continuous video EEG allows visuo-electrophysiological analysis and convergence. Video EEG is therefore considered the mainstay of localization. The usage of EEG offers the investigator a threefold advantage. [15] It allows a too further characterize the inter ictal EEG, to detect, characterize and quantify the patient's habitual seizures and to acquire physiologic data regarding seizure localization that can be correlated and compared with anatomic data obtained from neuroimaging. [16, 17]

\subsubsection{Continuous Video EEG}

This is typically done over 5-7 days. The procedure requires inpatient care and close observation. The first stage involves activating procedures such as sleep deprivation and cessation of Anti-Epileptic Drugs. The patient is in a room with continuous video and electrophysiologic recordings simultaneously progressing. This allows characterizing the seizure behaviour which allows correlation of ictal behaviour with EEG discharge, establishment of whether a patient has more than one seizure type, and finally lateralization and localization patterns of the seizure critical for further decision making. [15]

\subsubsection{Imaging Studies}

The most important subset of investigations regarding surgical planning involve imaging. MRI is the modality of choice. MRI epilepsy protocol which includes T1, T2 FLAIR, susceptibility weighted sequences are used commonly to detect lesions and regions of abnormal development, such as DNETs, cavernomas and gliomas. Hippocampal sclerosis is the most common pathology detected. fMRI, MRS, DTI, tractography are also important in surgical planning. Other imaging modalities of import include PET scan and SPECT.

i. Quantitative MR Imaging

In some patients with intractable focal epilepsy, structural MRI does not demonstrate a lesion. [21, 22]

Usually a 3D T1 weighted image offers an objective means of analysing MR images and presents an improved likelihood of detecting subtle lesions. Volumetric neocortical measurements may provide an objective way to evaluate the 
extent of resection and its relation to surgical results. This is especially true in early Mesial Temporal Sclerosis and subtle dysplasia of the cortex. (Figure 4) [23]

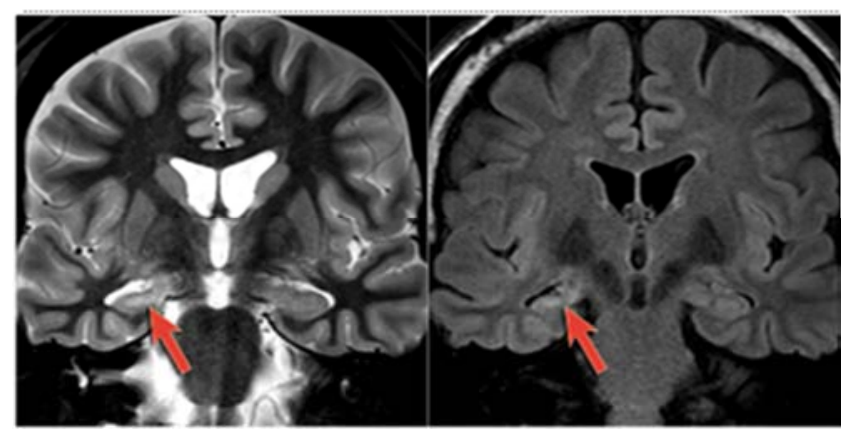

Figure 4. Quantitative MRI studies showing a coronal view of the brain in T2 \& FLAIR demonstrating volume loss \& gliosis in the right hippocampus as shown by the arrows.

\section{ii. DTI (Diffusion Tensor Imaging)}

Diffusion of substances soluble $n$ water presents an analysis of membrane integrity which differs from normal to abnormal. Diffusion is greatest parallel to the white matter tracts but minimal perpendicular to them. Hence Tractography enables the visualisation of tracts with respect to the lesion and allows accurate planning and deficit free outcomes for surgery.

DTI is useful in MCD and acquired lesions where there is increased mean diffusivity, increased perpendicular diffusivity. Intraoperative DTI is also available in on table MRIs where real time analysis of tract position is available. [24]

iii. MRS (Magnetic Resonance Spectroscopy)

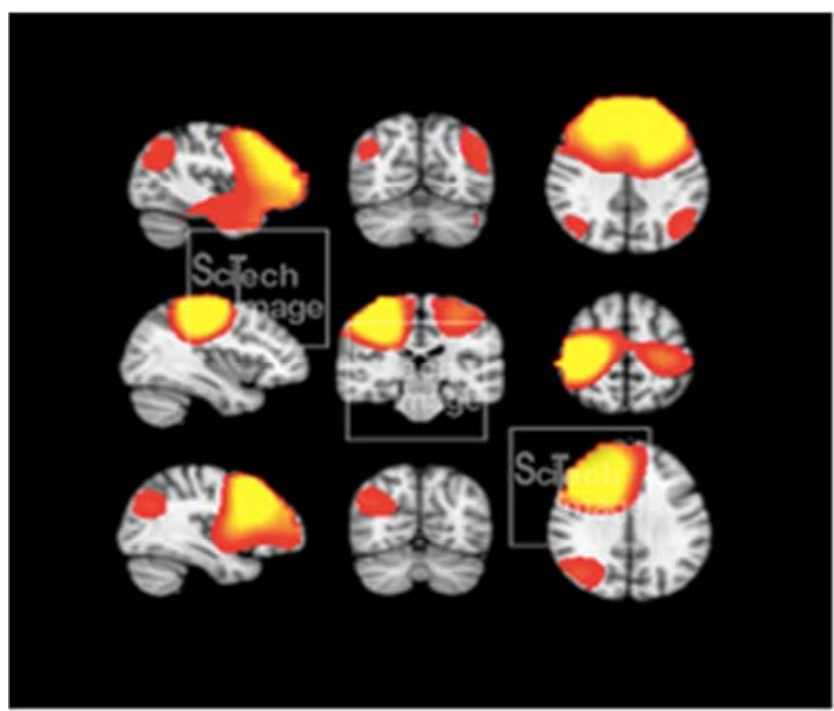

Figure 5. Showing functional MRI images in the brain describing hypermetabolic areas during an activity.

This is a newer adjunct to MRI. Here the altered concentration of NAA and Choline shows the nature of Lesional metabolism characteristic of certain lesions. The MR spectroscopy findings are consistent with the Histopathological characteristics of reduced neuron cell counts or neuronal dysfunction with increased glial cell numbers. This is of relevance in extra temporal lobe epilepsy, where the ability of MR spectroscopy to lateralize the epilepsy is less than $50 \%$. [24]

iv. fMRI (Functional MRI)

This is used for mapping language, memory and sensorimotor location for presurgical planning.

This imaging is based on the observation that increased neuronal activity is associated with increase in Cerebral Blood Flow and therefore an increase in the oxyhaemoglobin / deoxyhaemoglobin ratio, thus predicting active functioning areas of the brain over gliosis or quiescent areas. (Figure 5)

v. PET (Positron Emission Tomography)

Radio-labelled glucose (18FDG) to measure brain metabolism. Interictal PET usually shows hypometabolism in the seizure focus, especially in Temporal Lobe Epilepsy (TLE). Ictal PET is not practical due to the extremely short half-life of radiotracers used. PET is useful in MRI negative TLE, though it may be helpful in extra-temporal epilepsy as well. FDG PET identifies sites of interictal metabolism in $70 \%$ patients with TLE and $60 \%$ frontal lobe onset seizure with less than $5 \%$ false positivity. [23, 24] However, C -11 flumazenil PET effective in hippocampal sclerosis \& shows pathological foci in a more circumscribed fashion. (Figure 6)

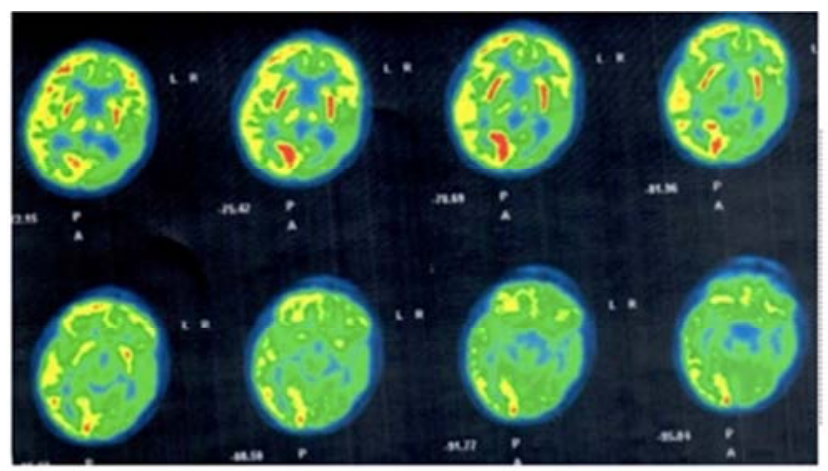

Figure 6. Showing images of the brain with areas of hypoperfusion shown in blue and areas of hyperperfusion shown in red.

\section{vi. SPECT (Single Photon Emission CT)}

Ictal SPECT is the ideal method for localization and lateralization of seizure onset. It shows hypermetabolism $\left({ }^{99} \mathrm{Tc}\right.$ HMPAO) with an $89 \%$ sensitivity for localization (Vs PET $63 \%$ ) SPECT also performed better in the setting of negative MRI, and false lateralization is rare. Post Ictal SPECT is comparable to interictal PET (70\%). Inter ictal scan shows hypoperfusion in a large area in the hemisphere of onset. Interictal scan though has less sensitivity for seizure, $50 \%$. Ictal / Interictal quantitative difference analysis provides for the best and most reliable information for seizure focus localization. [25, 26]

vii. (MEG) Magnetoencephalography

This is a rare non-invasive technique, which measures the magnetic field produced by the electric currents in the brain (epileptiform discharges). The subtle flux created between the magnetic field of the seizure discharge is crossed with an intense magnetic field produced in a confined specially prepared space to effect superb electrophysiological localisation. [25, 26] The electric points are superimposed 
onto an MRI) usually T1 weighted image) to allow structural and spatial localisation as well. The advantages are that it is a sensitive tool for localisation, where signals generated are not distorted by skull and scalp as in EEGs. The disadvantages are that it is a very expensive investigation and as mentioned earlier, the setup needs to be kept in a shielded room. to eliminate outside magnetic signals, which will interfere with the results. (Figure 7)

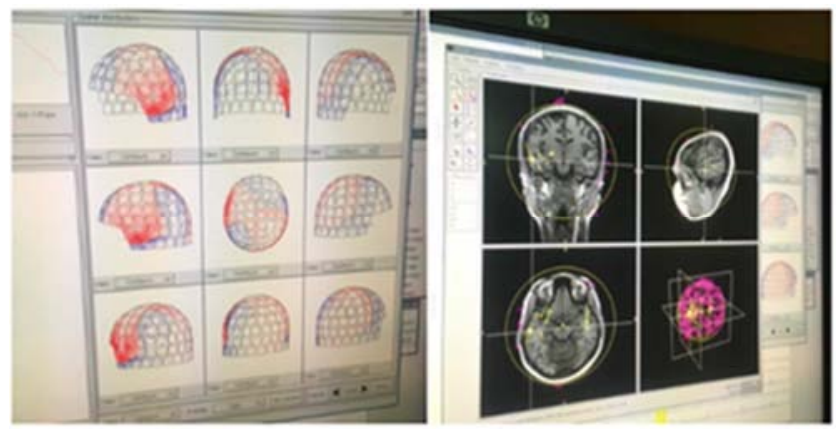

Figure 7. Showing the output of a MEG scan where the images show a clear localisation of the epileptic zone which in the second image is superimposed on a T1 weighted MRI image of the brain to aid surgical planning.

\subsubsection{Neuropsychological Testing}

These include Epilepsy related cognitive impairment, Memory status for baseline and Prediction of potential post op deficits, IQ testing -disparity in different components lateralise the EZ, along with Verbal IQ and performance IQ.

\subsection{Post Investigation Evaluation}

The analysis of the phase 1 investigations should lead to 1 of the 4 results.

a) Localization of seizures to one temporal lobe.

b) Bilateral localization.

c) Posterior temporal or extra temporal.

d) Inability to localize the site of seizure onset.

The patients in group 1-3 undergo further phase 2 investigations for optimal results during surgery. [25]

\subsection{Phase 2}

These investigations are invasive and done usually on the cortex after surgical dissection. The investigations allow complete or maximum safe resection of the causative Epileptic Zone (EZ).

The Goals are to confirm the primary EZ is the only focus, and to determine the risk involved post resection causing cognitive and neuropsychological deficits. Indications include situations where the seizure onsets are lateralized but not localized, the seizure onsets are localized but not lateralized, neither lateralized nor localized (Diffuse), dual pathologies, and finally to allow identification of the eloquent cortex. Advantages include allowing recording of seizure activity to be done from close proximity to the brain which is not possible with EEG bypassing the scalp and skull. The exact EZs are identified thus. Disadvantages include the fact that implantation involves a surgical risk. The different implements in phase 2 testing include. [25-27]

\subsubsection{Electrocorticography (ECoG) and Open Brain EEG}

This novel technique allows for measurement of electrophysiological activity on the brain surface. The electrodes used vary as stated below.

i. Epidural Electrodes

Electrodes are placed on the dura only. Advantages include a high amplitude EEG without muscle or movement artefact along with Good lateralization. The risk of infection is minor compared to more invasive methods. The disadvantages include the fact that it can record EEG changes only over the lateral convexities and gives only an approximate localization.

ii. Subdural Electrodes

These electrodes measure seizure activity on the surface of the cortex. These are of 3 types - Grid, Strip, and Foramen ovale electrodes. Strip electrodes are used for lateralization by placing them through burr holes and for localization with grid electrodes. Grid electrodes are much more intensive with the placement of a grid of electrodes onto the surface. The advantages are that no motion artefacts occur as it is fixed to dura, and that it does not penetrate the cerebral tissue. A wide area of cortical surface is covered hence making detection of the EZ easy. It can be used for brain mapping as well minimising post procedure deficits. [26]The disadvantages are all surgical effects such as haemorrhage, oedema and infection. No specific effects have been noted as yet. (Figure 8)

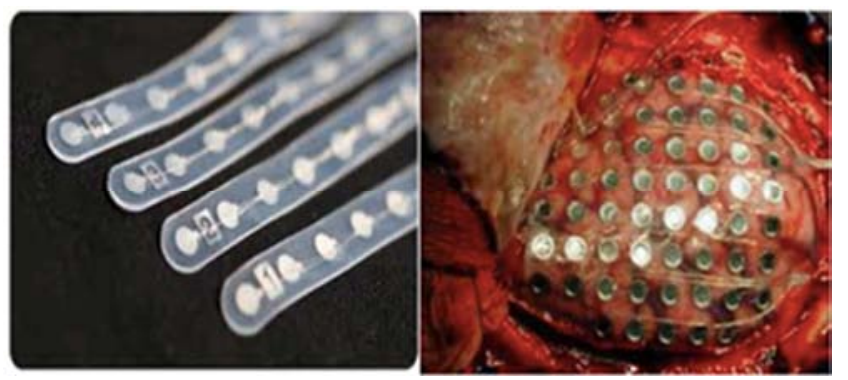

Figure 8. Showing strip and grid electrodes used for electrocorticography.

\section{iii. Foramen Ovale Electrodes}

These are Intra cranial electrodes introduced percutaneously into the foramen ovale using fluoroscopic guidance to measure electric spikes in the mesial temporal structures. It may be used for Mesial Temporal Lobe Epilepsy (MTLE) detection alone. The advantages are that the procedure involved is minimally invasive. However, as it measures only MTL biased information remote signals are not well recorded. Rarely complications of improper placement of the electrode under fluoroscopic techniques may lead to Facial pain, Temporo-Mandibular joint dysfunction, and mandibular hematomas. [26]

iv. Intra Cerebral Depth Electrodes

Flexible electrodes with multiple contact points are placed stereotactically at depths into the brain substance. The readings help to determine the depth of tissue needed for resection allowing no normal white matter to be damaged during resection. Common sites include the hippocampus, 
amygdala, orbitofrontal, cingulate gyri. Depth electrodes are indicated in Extra temporal EZ as well as with subdural electrodes in dual pathology.

\subsubsection{WADA Test}

Juhn Wada in 1960, at the Montreal Neurological Institute created a unique invasive test to detect dominance. The test is on historic importance now, yet is important $t$ understand the evolution of investigations to decipher dominance and speech control. The procedure involved injecting 150-200mg of Amobarbital (Amytal) into the common carotid artery (Prior to this and angiogram must be done to rule out persistent trigeminal, otic, hypoglossal or proatlantic arteries to avoid respiratory and circulatory problems as a result of shunting to the brainstem) The injection is made with the patient counting, the forearms up in the air and the fingers either moving constantly or gripping an examiner's hands. As the injection is completed, contralateral hemiplegia and flaccid limbs occur with one hand falling off or loosening its grip on the examiners hand. the hemiplegia lasts for 1.5 to 5 minutes. At this time speech is tested. If speech is normal, then the side injected is NON dominant. This is followed by dysphasia and lasts for 1-3 minutes following which normal speech. Functional MRI has now replaced the WADA test. Dangers include a 3\% possibility of permanent paralysis along with speech loss. [26, 27]

Once investigations are complete and the plan for surgery has been agreed upon, the plan is discussed with the patient and relatives by the multidisciplinary team to appraise them of all features including deficits which may occur post operatively and long term consequences of surgery and the disease. Five factors which are predictive for good seizure control are

1) Clear abnormality on MR images;

2) Absence of status epilepticus;

3) MR imaging-confirmed ganglioglioma or DNET;

4) Concordant lateralizing memory deficit; and

5) Absence of dysplasia on MR images

No significant differences exist regarding different resection types performed for comparable lesions. Neuropsychological testing done before surgery ensures better postoperative results after limited resections especially in attention level, verbal memory, and calculated total neuropsychological performance. [27]

\section{Surgical Procedures}

Surgical techniques range from curative to palliative procedures which minimise the morbidity of the disease making a significant improvement in the quality of life in a specific subset of patients. As shown in Figure 9, a variety of factors ranging from pathology, location, age and presence of eloquent cortex around the EZ serve as predictors of surgical outcome. [28]

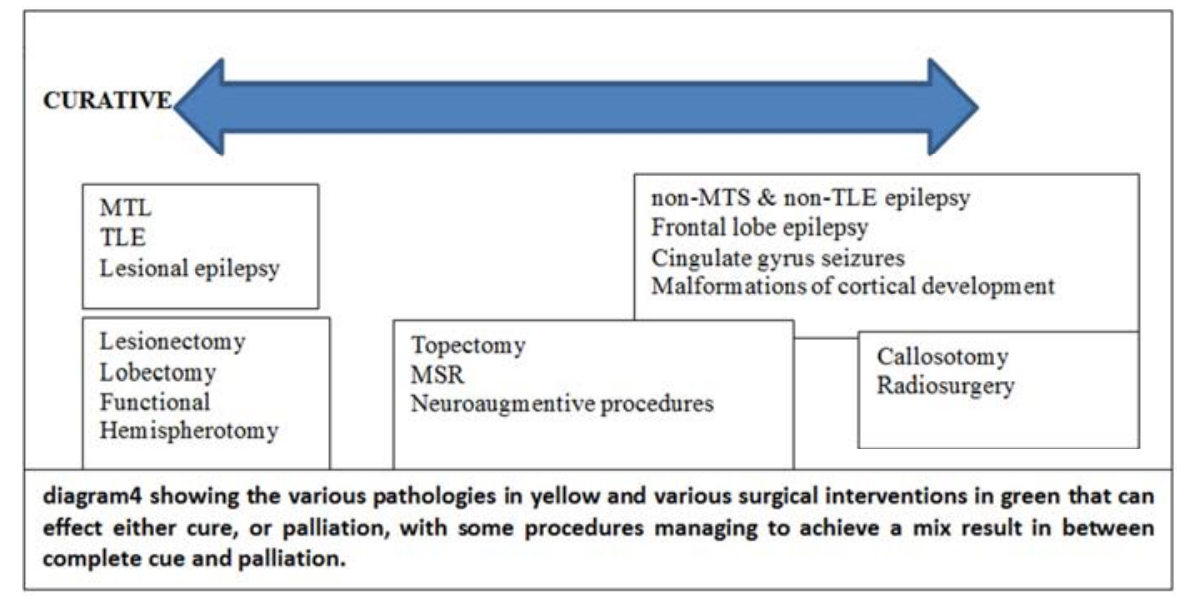

Figure 9. A variety of factors serve as predictors of surgical outcome.

The utility of temporal lobe surgery for intractable epilepsy vs. Continued treatment with antiepileptic drugs was analysed by Wiebe et al, NEJM 2001. The study was a prospective, randomized, controlled trial involving eighty patients randomized to surgery or medical treatment for one year. After a year, those undergoing surgery had a much higher rate of seizure freedom $(58 \%$ versus $8 \%)$, significantly better quality of life than the observation or medical category making epilepsy surgery highly recommended. These results have been replicated across many centres in different countries showing the increased utility and acceptance of epilepsy surgery over the years in treating medically intractable seizures in patients of all ages. We now present a few important aspects to select surgical techniques and their uses and concerns below. [24]

\subsection{Resective Procedures}

These include lobectomies, Lesionectomies, topectomies and other similar procedures. Surgery for temporal and extratemporal epilepsy is dealt with in brief below. Due to its extensive use, the temporal lobectomy along with its derivatives will be discussed in some detail.

\subsubsection{Temporal Lobe Surgery}

The techniques of Temporal Resection include:

1. Temporal Lobectomy.

2. Anterior temporal lobectomy (ATL).

3. Anteromedial temporal resection (AMTR).

4. Tailored (ECoG with or without speech mapping). 
5. Selective Medial Resection.

6. Selective Amygdalohippocampectomy.

a) Transcortical approach.

b) Trans-sylvian approach.

c) Subtemporal approach.

i. Anterior Temporal Lobectomy (ATL)

Here the anterolateral temporal lobe $(4-4.5 \mathrm{~cm}$ from temporal tip along middle temporal gyrus), amygdala, uncus, hippocampus and Parahippocampal gyrus to level of collicular plate are removed. Complications include hemiparesis which occurs upto $1.25 \%$ of cases, due to damage of the perforators to the anterior choroidal artery, contra lateral superior quadrantanopia from damage to the Meyer loop, infection, cranial nerve III or IV palsies seen in up to $20 \%$ of cases, and verbal memory problems particularly in speech-dominant temporal lobe resection. [23-26]

ii. Anteromedial Temporal Resection (AMTR)

Spencer et. al. in 1984 published the first description of this procedure. Here the anterior $3-3.5 \mathrm{~cm}$ of middle and inferior temporal gyrus, amygdala, $3-4 \mathrm{~cm}$ of hippocampus and the Parahippocampal gyrus are resected. Candidates for AMTR have complex partial seizures with semiology typical of mesial temporal lobe epilepsy. MRI evidence of unilateral hippocampal atrophy and an increased T2-weighted signal from the hippocampus area indicating neuronal loss, unilateral temporal lobe hypometabolism on PET scans and EEG confirmation that seizures begin over the temporal area ipsilateral to the hippocampal atrophy or PET scan evidence of hypometabolism in anteromedial temporal region. [22-24] (Figure 10)

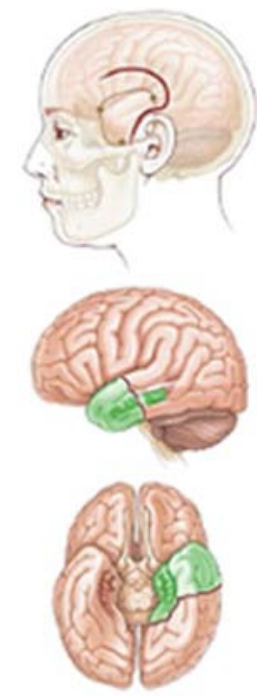

Figure 10. Showing the incision, and extent of resection for an anteromedial temporal lobectomy.

The procedure is a 2 stage surgery. The $1^{\text {st }}$ stage involves an anterior temporal lobectomy removing a major part of the temporal neocortex, and the $2^{\text {nd }}$ stage involving the removal of Parahippocampal gyrus, hippocampus and amygdala. Long term seizure outcome is excellent with initial seizure free rate at $80 \%, 5$ year seizure free rate at $50 \%$ Another reassuring feature of the procedure is the running down phenomenon, where incompletely resected EZs cause seizures initially but gradually loose the power to generate seizures, leading to better results over time. Cognitive outcomes are minimal in nondominant resections. The most common result involves neuropsychological deficits due to excision of the mesial temporal structures resulting in some memory loss. Health related quality of life is better after surgery. [26-28]

Specific complications include visual field loss due to injury to the geniculocalcarine fibres, contralateral superior quadrantanopia, contralateral homonymous hemianopia and horizontal \& vertical diplopia due to injury to the 3 rd \& 4th Cranial Nerves during resection. Hemiparesis may also occur due to anterior choroidal artery injury, basal ganglia injury during amygdala resection, injury to arteries in the hippocampal sulcus, and retraction injury of ipsilateral cerebral peduncle. Lastly aphasia may occur during dominant lobe resections due to damage to the superior temporal gyrus and the Wernicke's area. [26]

iii. Selective Amygdalo-hippocampectomy

This is a tissue sparing operation with removal of mesial temporal structures, the uncal portion of amygdala, anterior portion of hippocampus, and a portion of Parahippocampal gyrus. The approach may vary from Transcortical (via middle temporal gyrus as proposed by Niemeyer 1958) vs. Transsylvian as proposed by Yasargil in 1982 with a new Subtemporal approach proposed by Hori in 1991. The trans temporal approach through the middle temporal gyrus proposed by Niemeyer is a more direct approach but causes neocortical breach and injury [28].

The transylvian approach has a complete avoidance of neocortical injury but is more demanding and has a danger of causing injury to vascular structures in the sylvian fissure. The subtemporal approach is associated with fewer neuropsychological deficits but has a higher incidence of retraction injury deficits. (Figure 11)

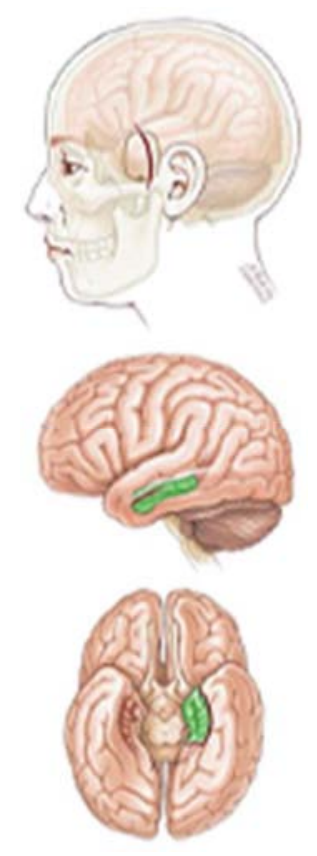

Figure 11. Showing the incision, and extent of resection for a selective amygdalohippocampectomy. 


\subsubsection{Extratemporal Epilepsy Surgery}

The techniques described here include:

1. Topectomy.

2. Lesionectomy.

3. Anatomical Hemispherectomy.

i. Topectomy

This is the resection of focal segment of the cerebral cortex. It is primarily used in Extra-Temporal epilepsy. The indications include medically intractable seizures interfering with development and mature EZs with no possibility of spontaneous regression. The procedure involves preservation of the essential motor and language areas followed by a subpial dissection and gyral resection done upto the bottom of sulcus, removing only grey mater.

Outcomes are encouraging with seizure free rates approaching $45 \%$.

ii. Lesionectomy

These are surgical resections aimed at curing epilepsy by removing structural brain lesions such as malformations of cortical development, low-grade neoplasms, vascular malformations, etc.

Surgical approach depends on lesion location. Intraoperative ECOG is generally used to delineate margins of epileptogenic zone and guide resection as well as evaluate completeness of resection. ECoG correlated significantly with clinical improvement. Its Sensitivity is approximately $100 \%$ $(95 \% \mathrm{CI} ; 96-100 \%)$ and specificity is $68.3 \%(95 \% \mathrm{CI}$; $51.8-81.4 \%$ ) with a positive predictive value of $89.9 \%$ The suspected regions of epileptogenesis may involve eloquent cortex, hence mapping of cortical function during diagnostic work-up using fMRI, MEG.

Coupled with mapping by intraoperative cortical stimulation assists in optimal seizure control as well as in minimising deficits. In the absence of pathological abnormalities, extratemporal resections represent the poorest outcome of all seizure surgery. [28]

iii. Hemispherectomy

Dandy performed the first hemispherectomy in 1923 for a diffuse malignant glioma. Today's indications are for seizures arising over most of one hemisphere, severe hemispheric damage during development processes, Sturge-Weber syndrome, extensive perinatal Infarcts, Hemimegaloencephaly, Rasmussen's Encephalitis and (increasingly rare nowadays but of historical value), failed functional hemispherectomy patients. The goal is to remove or disconnect all of cortex of one hemisphere from the rest of the brain. The steps involve a Trans sylvian approach to expose the circular sulcus. Temporo-mesial resection is followed by a complete opening of the lateral ventricle and Frontobasal and Posteromediobasal disconnection. In a resective procedure, all the disconnected hemisphere is removed piecemeal (Anatomical Hemispherotomy) [28].

An ideal candidate is one who already has contralateral hemiparesis, hemianopsia along with intractable seizures. In the short term seizure cure occurs in most patients. However progressive worsening of the neurological status of the patients a few years after surgery (an average of 8 years after surgery), leading to death in up to $30-40 \%$ of the patients occurs due to extensive iron deposits on the brain surface, with a membrane lying over the hemispherectomy cavity called "superficial cerebral hemosiderosis" This has led to a preference for functional Hemispherotomy which achieves the same seizure control rates of the resective counterpart without long term morbidity and mortality. [29]

\subsection{Disconnection Procedures}

Disconnection procedures are gaining importance in the treatment of wide areas of EZs effecting optimal seizure control with minimal resective surgery. It however does not entail, that the corresponding deficits will also be mild or minor. Depending upon the area disconnected, significant disability can be created, which needs to be discussed and explained before surgery to the relatives and patient in order to allow better post op recovery and acceptance. The various procedures include:

1. Corpus Callosotomy,

2. Multiple Subpial transections,

3. Posterior Quadrant disconnection, and

4. Functional Hemispherotomy,

We will go through a few prominent procedures here in this section. [27, 28]

\subsubsection{Callosotomy}

Introduced in 1940 by van Wagenen and Herren, it involves the transection of anterior third of the corpus callosum. The rationale is that disruption of rapid spread of certain seizures from one hemisphere to the other gives better seizure control and changes a generalised seizure into a partial seizure thereby improving morbidity. Seizures that respond well include Drop attacks, Atypical absence, and GTC seizures with diffuse EZs. The most important complication apart from those of surgery is disconnection syndrome. (Alien hand syndrome) which may be problematic post surgery especially for extensive disconnections. [23, 27, 29]

\subsubsection{Multiple Subpial Transections}

Developed to treat epilepsy arising from cortex that cannot be resected (either an eloquent area or a very wide region). The rationale implies that a cortical island wider than $5 \mathrm{~mm}$ or with horizontal connections larger than $5 \mathrm{~mm}$ is required to support paroxysmal discharge. Thus, disruption of horizontal connections within cortex that are vital for synchronizing neural activity, help in seizure control without affecting ascending and descending fibres. Thus, the procedure involves disconnecting the grey mater columns in the eloquent cortex. Indications include $\mathrm{EZ}$ in eloquent cortex (sensory, somatosensory, visual), associated focus on peroperative intra cranial investigations such as Electrocorticography (ECoG) or MRI, Landau Kleffner syndrome, and patches of EZs caused by Rasmussen's encephalitis. The procedure involves a wide craniotomy and isolating the EZ with ECoG using grid \& strip electrodes, followed by transections in the pia and grey mater done for 5-6 $\mathrm{mm}$ a piece all around separating the EZ from the rest of 
the grey matter. Care is taken to avoid vascular injury.

Post-Surgery, ECoG is repeated to ensure all the EZs are within the resected region and to confirm reduced IED's. Complications include transient deficits restricted to the eloquent cortex transected, sane some Sub Arachnoid Haemorrhage. The Outcomes are usually good with seizure free rates as high as $90 \%$. [27, 29]

\subsubsection{Functional Hemispherectomy \& Posterior Quadrant Disconnection}

Described initially in the 1970 s by Rasmussen, the procedure attained popularity over the years owing to the efforts of 2 French neurosurgeons in the 1990s. 2 different approaches were described, a vertical approach was described by Delalande and colleagues while a lateral approach was described by Villemure et al. The common goal of all of the Hemispherotomy is the interruption of the corpus callosum, the internal capsule and corona radiata, the mesial temporal structures and lastly the frontal horizontal fibres. The standard functional hemispherectomy involves 4 steps, Temporal lobectomy including amygdalohippocampectomy, Suprasylvian central block disconnection, Transventricular callosotomy (including the splenium) along with Frontal and parieto-occipital disconnections. [28, 29] A smaller version involving only the posterior quadrant and sparing the frontal connections has been popularised as the Posterior Quadrant Disconnection. This is popular in EZs isolated to the posterior quadrant and is less morbid than the complete Hemispherotomy. (Figure 12)

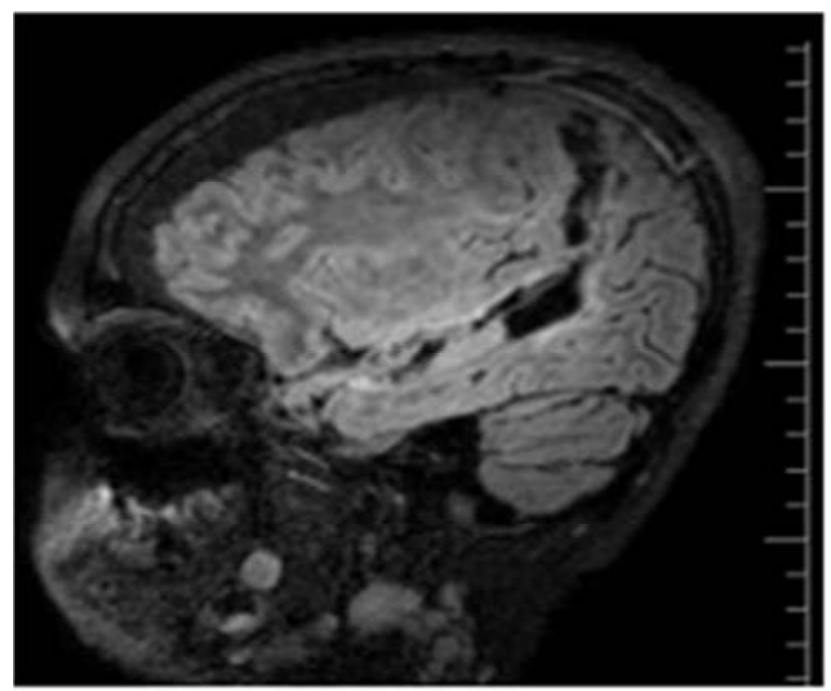

Figure 12. Showing the post op MRI of a posterior quadrant disconnection demonstrating the region disconnected from the rest of the functioning brain.

Rasmussen's study in 2007 involved a total of 19 patients between 2001 and 2007 which were followed up for 198weeks after surgery. He reported Engel's class I outcomes in 18 patients and class II in 1 patient. There were deficits which improved well. Postoperative limb power improved in 3 patients while the rest had the same power and movement as preop. Cognitive functions improved in all patients as well.

\subsection{Neuroaugmentive Procedures}

These include Deep brain stimulation of various subcortical nuclei and vagal nerve stimulation techniques. These are described below.

\subsection{1. (VNS) Vagal Nerve Stimulation}

FDA approval of VNS was received in 1997 as an adjunctive therapy in patients 12 years of age and older for intractable epilepsy of all kinds. The mechanism of action of VNS not clear. It is thought that desynchronizing electroencephalography activity leads to relief from the physical activity of the seizure thereby effecting remission. Indications for VNS includes a failure of medical therapy \& the patient being unsuitable for resection. In the procedure, a standard pacemaker generator which houses a lithium battery along with electronics are implanted in a subclavicular pocket. The lead wire is tunnelled into the left carotid sheath via a transverse or longitudinal neck incision. The spiral endings of the leads attached to left vagus nerve. The left vagus is used due to a lower percentage of efferent fibres to the atrioventricular node, thereby sparing the heart from arrythmias that may occur during stimulation. Seizure Control is well documented. Here $35-45 \%$ of patients have decreased frequency of seizures exceeding 50\% [27-29].

And around $2 \%$ become seizure free. The complications include infection $(5-7 \%)$, vocal cord paralysis $(\sim 1 \%$ of patients), hoarseness, cough, dyspnoea, nausea, and rarely obstructive sleep apnoea.

\subsubsection{Deep Brain Stimulation (DBS) for Epilepsy}

General concept here is to disrupt the seizure generating network in order to prevent initiation of seizures or terminate seizures underway. The target of stimulation has undergone discussion and revision over the years. These include the cerebellar nuclei which cause inhibition of thalamic nuclei by modulating the activity of efferent cerebellar nuclei and more importantly, the Anterior nucleus of the Thalamus, which is the central relay station of the limbic system and is closely connected both to the hippocampi as well as to extensive areas of the neocortex [29].

Subthalamic nucleus and Caudate nucleus. Anticonvulsive mechanisms range from inactivation of the neurons by blocking depolarization, reduction of the recruitability of neurons on the basis of the rhythmic activity they induce, activation of inhibiting neurons and their projections, and finally effecting changes in the properties of networks (desynchronization, antikindling effects).

Stimulation Paradigms can either by Open Loop or Closed Loop in their activity. Open loop stimulation involves continuous or cyclical stimulation, while in Closed loop stimulation, electrical stimulus is delivered in response to the onset of a seizure There is also Patient-activated stimulation, where stimulation is initiated by the patient or caregiver when they feel or see a seizure. Complications include infection rates of $6.1 \%$, misplacement of electrodes (upto 4.4\%), $0.5 \%$ to $1 \%$ incidence for symptomatic bleeding, electrode breakage in at $1.8 \%$ of cases and Skin ulcerations in $1.3 \%$ of patients. 
[29]

\subsection{Radiosurgery for Epilepsy}

Why radio surgery? While selective temporal resections are effective, morbidity is low, but not zero. Infection, neuropsychological changes, blood loss (intra-operative, post-operative) and other focal neurological deficits which maybe be possible during and after surgery make the post-operative recovery difficult. Medical contraindications of open surgery which are a major obstacle in certain disease coupled with some patients fear of surgery, make the prospect of radiosurgery appealing. There is however only an indirect evidence for efficacy is seizure control as demonstrated in Tumors, Hypothalamic hamartomas and in AVMs. Neurons themselves are resistant to radio necrosis. However, vasculature and glia are sensitive.

Endothelial damage due to radiation especially to small blood vessels leads to astrocytic reactions. Eventual neuronal damage results from ischemia caused by vascular inflammation.

Other hypotheses suggest that irradiated neuronal circuits undergo neuromodulation that renders an anticonvulsant (or, sometimes, a paradoxically proconvulsant) reaction.

Radiation directed at specific sites to maximise efficacy. The regions include the temporal portion of the amygdala, the anterior $2 \mathrm{~cm}$ of hippocampus and adjacent Para hippocampal gyri.

The total volume within the $50 \%$ isodose line is between 5.5 and $7.5 \mathrm{cc}$. A dose of $20-24 \mathrm{~Gy}$ to the $50 \%$ isodose line is given either directly or in fractions. Complications of radiosurgery include initial increase in auras, headaches, visual field deficits ( $52 \%$ of patients - mostly quadrantanopia), and lastly a long waiting period for the effects of radiation to occur.

\section{Surgical Outcome}

The outcome of seizure control post surgery is measured by the Engels score shown below.

Engel's classification of postoperative outcome

Class I: Free of disabling seizures

A: Completely seizure free since surgery

B: Nondisabling simple partial seizures only since surgery

C: Some disabling seizures after surgery, but free of disabling seizures for at least 2 years

D: Generalized convulsions with AED discontinuation only Surgical outcome

Class II: Rare disabling seizures ('almost seizure free')

A: Initially free of disabling seizures but has rare seizures now

B: Rare disabling seizures since surgery

$\mathrm{C}$ : More than rare disabling seizures since surgery, but rare seizures for the last 2 years

D: Nocturnal seizures only

Class III: Worthwhile improvement

A: Worthwhile seizure reduction

B: Prolonged seizure-free intervals amounting to greater than half the followed-up period, but not $<2$ years
Class IV: No worthwhile improvement

A: No Significant seizure reduction

B: No appreciable change

C: Seizures worse

\section{Predictors of Recurrence}

These include:

1. Age at onset of epilepsy: earlier age (usually $<5$ years), have a favourable postoperative outcome.

2. Duration of epilepsy: long history correlates with worse outcome.

3. Age at surgery: Hippocampal Sclerosis patients aged $\leq 24$ years were about four times more likely to be seizure free at 5 postoperative years when compared with the older surgical group (36 years or older).

4. Type of disease involved: Lesional epilepsy has a better outcome than idiopathic epilepsy.

5. Absence of secondarily generalized tonic-clonic seizures (GTCs): $57 \%$ of MTLE-HS patients with SGTCS have a 1 -year remission compared to $80 \%$ remission rate in those who had only partial seizures. Patients who had no GTCs were 2.2 times more likely to be seizure free 5 years after surgery.

6. Preoperative seizure frequency: Seizures ( $>20 /$ month) were associated with lower rates of seizure freedom.

7. History of febrile seizures: Simple febrile convulsions, have a favourable prognostic value for surgery. [29]

\section{Conclusion}

Appropriate patient selection with a judicious use of technology as well as sound clinical acumen coupled with a multidisciplinary approach can lead to excellent results in drug recalcitrant epilepsy. Further advances have improved outcomes and reduced risks and complications. A case by case basis using the axioms discovered and postulated can lead to even better outcomes for patients and researcher alike.

\section{References}

[1] Maton BM, Kuniecky RI: Proton MRS: N-acetyl aspartate, creatine, and choline. Adv Neurol 83: 253-259; 2000.

[2] Devinsky O, Pacia S: Epilepsy surgery. Neurology clinics 11: 951-971; 1993.

[3] Szelies B, Pawlik G et al: MRI guided flumazenil and FDG PET in temporal lobe epilepsy, Neuroimage 3: 109-118; 1996.

[4] Ho SS, Berkovic SF et al: Comparison of Ictal SPECT and interictal PET in the presurgical evaluation of temporal lobe epilepsy, Annals of neurology 347: 738-745; 2000.

[5] Wiebe et al: Utility of temporal lobe surgery for intractable epilepsy vs. Continued treatment with antiepileptic drugs, NEJM 12: 131-137; 2001. 
[6] Spencer et al: Anteromedial Temporal Resection, NEJM 10: $123-132 ; 2006$.

[7] Clusmann H, Schramm j, Kral T et al: Prognostic factors and outcome after different types of resection for temporal lobe epilepsy. J. Neurosurg. 97: 1131-1141; 2002.

[8] Tripathi M, Garg A, Gaikwad CS, et al: Intra operative electrocorticography in Lesional epilepsy. World Neurosurgery 19: 1222-1245; 2009.

[9] Oppenheimer DR, Griffith HB: Persistent intracranial bleeding as a complication of hemispherectomy. J Neurol Neurosurg Psychiatry 29: 229-240, 1966.

[10] Sarat Chandra P, Padma Vasantha M, Gaikwad S et al: Hemispherotomy for intractable epilepsy. World Neurosurgery. 23: $234-241 ; 2008$

[11] Murphy M, Smith PD, Wood M, et al. Surgery for temporal lobe epilepsy associated with mesial temporal sclerosis in the older patient: a long-term follow-up. Epilepsia 2010; 51: 10249.

[12] Dunkley C, Kung J, Scott RC, et al. Epilepsy surgery in children under 3 years. Epilepsy Res 2011; 93: 96-106.

[13] Téllez-Zenteno JF, Dhar R, Wiebe S. Long-term seizure outcomes following epilepsy surgery: A systematic review and meta-analysis. Brain 2005; 128: 1188-98.

[14] Sperling MR, Feldman H, Kinman J, et al. Seizure control and mortality in epilepsy. Ann Neurol 1999; 46: 45-50.

[15] Choi H, Sell RL, Lenert L, et al. Epilepsy surgery for pharmacoresistant temporal lobe epilepsy: a decision analysis. JAMA 2008; 300: 2497-505.

[16] Wiebe S, Jette N. Epilepsy surgery utilization: Who, when, where, and why? Curr Opin Neurol 2012; 25: 187-93.

[17] Hamiwka LD, Macrodimitris S, Tellez-Zenteno J, et al. Social outcomes after temporal or extra-temporal epilepsy surgery: a systematic review. Epilepsia 2011; 52: 870-9.

[18] 35. Wiebe S, Gafni A, Blume WT, et al. An economic evaluation of surgery for temporal lobe epilepsy. J Epilepsy 1995; 8: 227-35.

[19] Bowen JM, Snead OC, Chandra K, et al. Epilepsy care in Ontario: an economic analysis of increasing access to epilepsy surgery. Ont Health Technol Assess Ser 2012; 12: 1-41.
[20] Téllez-Zenteno JF, Hernández Ronquillo L, Moien-Afshari F, et al. Surgical outcomes in lesional and non-lesional epilepsy: a systematic review and meta-analysis. Epilepsy Res 2010; 89: 310-8.

[21] Téllez-Zenteno JF, Dhar R, Hernández-Ronquillo L, et al. Long-term outcomes in epilepsy surgery: antiepileptic drugs, mortality, cognitive and psychosocial aspects. Brain 2007; 130: 334-345.

[22] Spencer SS, Berg AT, Vickrey BG, et al. Health-related quality of life over time since resective epilepsy surgery. Ann Neurol 2007; 62: 327-34

[23] Hader WJ, Tellez-Zenteno J, Metcalfe A, et al. Complications of epilepsy surgery - a systematic review of focal surgical resections and invasive EEG monitoring. Epilepsia 2013; 54: $840-7$.

[24] Sherman EM, Wiebe S, Fay-McClymont TB, et al. Neuropsychological outcomes after epilepsy surgery: systematic review and pooled estimates. Epilepsia 2011; 52: 857-69.

[25] LangUtt JT, Westerveld M, Hamberger MJ, et al. Worsening of quality of life after epilepsy surgery: effect of seizures and memory decline. Neurology 2007; 68: 1988-94 Labiner DM, Bagic AI, Herman ST, et al. Essential services, personnel, and facilities in specialized epilepsy centers - revised 2010 guidelines. Epilepsia 2010; 51: 2322-33.

[26] Berg AT, Berkovic SF, Brodie MJ, et al. Revised terminology and concepts for organization of seizures and epilepsies: report of the ILAE Commission on ClassiUcation and Terminology, 2005-2009. Epilepsia 2010; 51: 676-85.

[27] Cross JH, Jayakar P, Nordli D, et al. Proposed criteria for referral and evaluation of children for epilepsy surgery: recommendations of the Subcommission for Pediatric Epilepsy Surgery. Epilepsia 2006; 47: 952-9.

[28] Helmstaedter C, Kurthen M, Lux S, et al. Chronic epilepsy and cognition: a longitudinal study in temporal lobe epilepsy. Ann Neurol 2003; 54: 425-32 Jette N, Quan H, Tellez-Zenteno JF, et al. Development of an online tool to determine appropriateness for an epilepsy surgery evaluation. Neurology 2012; 79: 1084-93.

[29] Haneef Z, Stern J, Dewar S, et al. Referral pattern for epilepsy surgery after evidence-based recommendations: a retrospective study. Neurology 2010; 75: 699-704. 